\title{
50 Jahre Bundeswehr, 50 Jahre »Innere Führung «: Anlass zu Reflexion und Reform
}

\author{
Kommission »Europäische Sicherheit und Zukunft der Bundeswehr« am IFSH
}

1. Die Bundeswehr feiert ihr 50-jähriges Bestehen. 50 Jahre Bundeswehr bedeuten auch 50 Jahre »Innere Führung «. Mit diesem Konzept sollte, die Ergebnisse der deutschen Geschichte vor Augen, der traditionelle Gegensatz zwischen dem Militär und einer demokratisch-pluralistischen Gesellschaft aufgehoben und die Geltung staatlicher und gesellschaftlicher Werte und Normen auch in den Streitkräften sichergestellt werden. Das visionäre Leitbild dieser Militärreform von Wolf Graf Baudissin seit dem Jahr 1950 konzipiert war der »Staatsbürger in Uniform«.

2. Von Anfang an jedoch erfuhr diese Reform grundsätzliche Ablehnung durch traditionalistische Gegenpositionen in der Bundeswehr. Der so genannte Gründungskompromiss von Himmerod im Oktober 1950 sah daher auch schon massive substanzielle Abstriche an der ursprünglichen Intention vor; der Reform kam eigentlich nur noch eine Randbedeutung zu. Entsprechend ambivalent verlief die weitere Geschichte der Bundeswehr. Heute, ein halbes Jahrhundert später, muss man alles in allem erkennen, dass der ursprüngliche Anspruch nur unvollständig eingelöst werden konnte und in ganz wesentlichen Punkten sogar gescheitert ist. Das Verständnis von Innerer Führung ist in der Bundeswehr häufig beliebig auf allen Ebenen. Zumeist wird sie auf eine rein formale, zwischenmenschliche Führungs- und Motivationstechnik reduziert. Indes, für eine entwickelte Demokratie ist das zu wenig.

3. Die deutschen Streitkräfte haben gerade in den letzten Jahren viele Veränderungen erfahren. Die Innere Führung jedoch kam dabei zu kurz, obwohl sie für die demokratische Integration der Bundeswehr von zentraler Bedeutung ist. Sie besitzt noch immer nicht den Stellenwert, den sie ursprünglich haben sollte. Daher ist heute wieder eine grundlegende und umfassende Militärreform notwendig, um Norm und Realität in eine neue Balance zu bringen.

4. Der »Staatsbürger in Uniform« ist auch heute kaum mehr als eine Fiktion, denn Selbstbestimmung und Eigenverantwortung, die dieses Leitbild impliziert, sind im militärischen Alltag nach wie vor eher unterbewertete und im Grunde ungeliebte, wenn nicht unerwünschte Eigenschaften. Stattdessen setzt man weiterhin auf zwangsrekrutiertes, zwangskaserniertes, mit zahlreichen antiquierten Normen traktiertes und bevormundetes Personal, das man »disziplinieren « und »erziehen« zu müssen glaubt. Die Kommission empfiehlt daher eine Umkehr auf allen Feldern der Personalpolitik und die Abschaffung der Wehrpflicht als ersten wichtigen Schritt zur Überwindung dieses antiquierten Soldatenbildes.
5. Politische Bildung in den Streitkräften stellt ein zentrales Element der Inneren Führung dar. Sie zielt darauf ab, das demokratische Selbstverständnis der Frauen und Männer in Uniform zu entwickeln und zu fördern, die unbedingte Beachtung der Menschenrechte sowie des Völker- und Kriegsvölkerrechts zu unterstützen, Respekt vor anderen Kulturen zu vermitteln, das Verständnis von Konfliktlagen zu erweitern und vieles mehr. Insofern begründet sie ganz wesentlich das soldatische Selbstverständnis mit. Indes wird seit Jahren, wenn nicht Jahrzehnten der desolate Zustand der Politischen Bildung in der Bundeswehr beklagt. Die Folgen zeigen sich unter anderem darin, dass insgesamt sowohl im Offiziers- als auch im Unteroffizierskorps der Bundeswehr ein bemerkenswerter Mangel an staatsbürgerlicher Allgemeinbildung und politischer Urteilskraft anzutreffen ist. Dies manifestiert sich in der weiten Verbreitung nationalistischen, konservativ-reaktionären Gedankengutes, der unreflektierten Huldigung des Kämpferkults bis hin zu rechtsradikalen Vorfällen. Daher ist die Politische Bildung in der Bundeswehr dringend zu verbessern. Da sie speziell im Hinblick auf die neue Rolle und Funktion der Bundeswehr für die Auftragserfüllung entscheidend ist, muss ihr der gleiche Stellenwert beigemessen werden wie der übrigen Einsatzausbildung. Die Kommission empfiehlt daher, den für die Politische Bildung vorgesehenen Zeitund Ressourcenansatz erheblich zu erhöhen.

6. Unter allen militärischen Normen ist das Prinzip von Befehl und Gehorsam wohl die schwerwiegendste Beeinträchtigung individueller Freiheitsrechte und demokratischer Werte. Zwar leuchtet es unmittelbar ein, dass militärische Effektivität nicht auf Befehl und Gehorsam verzichten kann; gleichwohl muss die Frage gestellt werden dürfen, ob seine heutige Ausgestaltung auch wirklich immer notwendig und gerechtfertigt ist. Wer dieses Prinzip als Tabu betrachtet, nimmt von vornherein in Kauf, dass Innere Führung Makulatur bleibt. Der Einwand, das geltende Befehlsrecht müsse schon deshalb unantastbar bleiben, weil es im militärischen Einsatz schließlich um Leben und Tod gehe, ist zu undifferenziert und kann nicht überzeugen. Auch Gehorsam war schon oft tödlich, und es ist eine keineswegs leicht zu beantwortende Frage, wann in der Geschichte des Militärs Gehorsam oder Ungehorsam das größere Unglück war. Es muss sich auch innerhalb von Streitkräften die Einsicht durchsetzen, dass die Berufung auf einen Befehl allein als Rechtfertigung für das eigene Handeln niemals ausreichend ist. Das Urteil des Bundesverwaltungsgerichts vom Juli dieses Jahres, das die Gewissensfreiheit des Soldaten über seine Gehorsamspflicht stellt, bestärkt uns in dieser Auffas- 
sung. Die Kommission empfiehlt daher, das anachronistische Befehlsrecht für die Bundeswehr zu modernisieren, Vorgesetztenbefugnisse sachgemäß zu beschneiden und dabei auch demokratiekonformere Einzelregelungen europäischer Partner zu übernehmen: Zum Beispiel sollten wie etwa in den britischen, französischen und niederländischen Streitkräften sämtliche unrechtmäßigen Befehle für die Soldatinnen und Soldaten stets auch unverbindlich sein.

7. Auch das Personalauswahlsystem der Bundeswehr legte in den vergangenen Jahrzehnten aus demokratischer Perspektive manche gravierenden Defizite offen. Abwertende Äußerungen von hohen und höchsten Militärs über das Konzept der Inneren Führung ziehen sich wie ein roter Faden durch die 50-jährige Geschichte der Bundeswehr - eine Provokation für jeden Demokraten. In den 1960er Jahren verkündete der damalige »Beauftragte des Generalinspekteurs für Erziehung und Ausbildung «, Freiheit und Demokratie seien »keine letzten Werte«. Und erst kürzlich bewertete der Kommandeur des »Kommandos Spezialkräfte« die Innere Führung als »unglückliche Konstruktion «. Selbst wenn man unterstellt, dass diese Generale nicht gerade wegen, sondern trotz derartiger Einstellungen in ihre Ämter gelangt sind, so bleiben solche Positionen inakzeptabel. Die Kommission empfiehlt daher, der Inneren Führung einen signifikant höheren Stellenwert im Ausbildungs- und vor allem im Auswahlsystem der deutschen Streitkräfte einzuräumen.

8. Meinungsfreiheit ist nach Auffassung des Bundesverfassungsgerichts »als unmittelbarster Ausdruck der menschlichen Persönlichkeit in der Gesellschaft eines der vornehmsten Menschenrechte überhaupt«. Das gilt auch für die militärische Binnenkultur. Der Grad der Integration der Bundeswehr in die demokratische Staats- und Gesellschaftsordnung spiegelt sich demnach auch darin wider, inwieweit öffentlich geführte Debatten auch in der Bundeswehr ihren Platz haben. Je mehr Soldatinnen und Soldaten über essenzielle Themen der Sicherheit in gleichermaßen kontroverser Manier diskutieren wie die zivile Öffentlichkeit auch, desto eher ist die Bundeswehr in die kommunikative Lebenswelt aller Bürger integriert. Umgekehrt gilt, dass Versuche der politischen Leitung und militärischen Führung, von oben Homogenität und Einheitlichkeit der Äußerungen von Soldatinnen und Soldaten in der Öffentlichkeit zu verordnen, dort eher Misstrauen erregen, den Verdacht erwecken, etwas verheimlichen oder das Publikum hinters Licht führen zu wollen, und somit die Institution des Militärs eher als Fremdkörper in der demokratisch-pluralistischen Gesellschaft erscheinen lassen. Als Lackmustest für die demokratische Reife und politische Kultur der Institution Bundeswehr erweist sich der Umgang mit Kritikern gerade innerhalb der Streitkräfte. Meinungsfreiheit darf hier nicht missinterpretiert werden als die Freiheit, öffentlich die Meinung des Bundesministeriums der Verteidigung vertreten zu dürfen. Daher fordert die Kommission, dass jeder Angehörige der Bundeswehr als Indikator für deren demokratisches, liberales Selbstverständnis ohne Angst vor Repressalien auch abweichende Positionen vertreten kann.

9. Schließlich könnte noch die Frage gestellt werden, ob seinerzeit der Begriff »Staatsbürger in Uniform « wirklich ganz glücklich gewählt worden ist oder nicht bereits selbst eine falsche Akzentuierung enthält. Dass Soldatinnen und Soldat eine spezifische Arbeitskleidung tragen und im Einsatz als Kombattanten erkennbar sein müssen, ist aus demokratietheoretischer Perspektive nicht gerade von alles überragender Bedeutung. Im Sinne der Inneren Führung ist vielmehr auch der Soldat Staatsbürger ohne Abstriche, „Staatsbürger ohne Wenn und Aber .

10. Innere Führung bleibt eine permanente politische Aufgabe; sie ist Bestandteil des demokratischen Prozesses. In der Demokratie herrscht in dieser Hinsicht immer Handlungsbedarf für den verantwortlichen Minister, das Parlament, den Wehrbeauftragten. Innere Führung braucht ein stärkeres Gewicht innerhalb des Bundesverteidigungsministeriums. Sie kann auch nicht durch die Filter verschiedener militärischer Hierarchieebenen hindurch effektiv wahrgenommen werden, sondern muss strukturell durchgreifend angelegt sein. Die Kommission fordert daher nachdrücklich die Bestellung einer bzw. eines mit den dazu erforderlichen Befugnissen ausgestatteten Beauftragten für Innere Führung im Verteidigungsministerium als deutliches politisches Signal für das auch nach 50 Jahren notwendige Bemühen um mehr Demokratie in den deutschen Streitkräften. 